\title{
ORTHOLOGIC DESARGUES' FIGURE and SPECIAL PAIRS OF SEMI-BILOGIC AND BILOGIC TETRAHEDRA: CORRIGENDUM
}

\author{
SAHIB RAM MANDAN
}

(Received 16 June 1980)

While going through either of my two papers entitled: 'Orthologic Desargues" figure' and 'Special pairs of semi-bilogic and bilogic tetrahedra' in J. Austral. Math. Soc. (Series A) 28 (1979), 295-302 and 303-308, see Fig. 2 in the other.

Flat 19, 'Vijaya', Chhedanagar

Bombay -400089

India 